\title{
Mutational analysis of the tuberous sclerosis gene TSC2 in patients with pulmonary lymphangioleiomyomatosis
}

Aristotelis Astrinidis, Leena Khare, Thomas Carsillo, Teresa Smolarek, Kit-Sing Au, Hope Northrup, Elizabeth Petri Henske

\begin{abstract}
Pulmonary lymphangioleiomyomatosis (LAM) is a rare disorder limited almost exclusively to women of reproductive age. LAM affects about $5 \%$ of women with tuberous sclerosis complex (TSC). LAM also occurs in women who do not have TSC (sporadic LAM). TSC is a tumour suppressor gene syndrome characterised by seizures, mental retardation, and tumours in the brain, heart, and kidney. Angiomyolipomas, which are benign tumours with smooth muscle, fat, and dysplastic vascular components, are the most common renal tumour in TSC. Renal angiomyolipomas also occur in $63 \%$ of sporadic LAM patients. We recently found that $54 \%$ of these angiomyolipomas have TSC2 loss of heterozygosity, leading to the hypothesis that sporadic LAM is genetically related to TSC. In this study, we screened DNA from 21 women with sporadic LAM for mutations in all 41 exons of TSC2. Twelve of the patients had known renal angiomyolipomas. No TSC2 mutations were detected. We did find three silent TSC2 polymorphisms. We conclude that patients with sporadic LAM, including those with renal angiomyolipomas, do not have a high frequency of germline mutations in the coding region of TSC2.

(F Med Genet 2000;37:55-57)
\end{abstract}

Keywords: TSC2; pulmonary lymphangioleiomyomatosis

Department of

Molecular Genetics,

Biochemistry, and

Microbiology,

University of

Cincinnati, Cincinnati,

OH, USA

T Smolarek

Division of Medical Genetics, Department of Pediatrics, The University of Texas Medical School, Houston, TX, USA $\mathrm{K}-\mathrm{S} \mathrm{Au}$

H Northrup

Correspondence to: Dr Henske

Revised version received 19 July 1999

Accepted for publication

30 July 1999

Pulmonary lymphangioleiomyomatosis (LAM) is a rare and often fatal disease of unknown aetiology affecting almost exclusively young women. ${ }^{2}$ Microscopically, LAM is characterised by diffuse infiltration of the pulmonary interstitium by smooth muscle cells and cystic distortion of the lung architecture. LAM can occur as an isolated disorder (sporadic LAM) or in association with tuberous sclerosis (TSC). TSC is an autosomal dominant disorder characterised by seizures, mental retardation, and hamartomatous tumours of the brain, heart, kidney, lung, and skin. LAM affects $2.3 \%$ of people (or $4.6 \%$ of women) with tuberous sclerosis. ${ }^{3}$ Renal angiomyolipomas, which are benign tumours composed of fat, smooth muscle, and dysmorphic vessels, occur in $70 \%$ of TSC patients and in $63 \%$ of women with sporadic LAM. ${ }^{4-6}$ The occurrence of angiomyolipomas in both TSC associated and sporadic LAM has led to the hypothesis that these diseases have a common genetic basis. ${ }^{4}$

There are two TSC genes, TSC1 on chromosome $9 \mathrm{q} 34^{8}$ and TSC 2 on chromosome $16 \mathrm{p} 13 .{ }^{9}$ The frequency of TSC 1 or TSC 2 loss of heterozygosity ( $\mathrm{LOH})$ in TSC associated angiomyolipomas is approximately $60 \% .^{10} \mathrm{In}$ contrast, TSC 2 LOH occurs in only $10 \%$ of angiomyolipomas from patients who do not have either TSC or LAM. ${ }^{11}$ We recently found that $54 \%$ of angiomyolipomas from women with sporadic LAM have LOH in the TSC2 region of chromosome $16 \mathrm{p} 13 .{ }^{12}$ We did not find TSC1 LOH in the sporadic LAM associated angiomyolipomas. ${ }^{12}$

The TSC2 LOH in the LAM associated angiomyolipomas led us to test the hypothesis that some patients with sporadic LAM have de novo germline mutations in TSC2 associated with a mild phenotype affecting only lung and kidney. Phenotypic variability in TSC is common, with some patients having only mild signs or symptoms of disease, and approximately $70 \%$ of TSC patients have new germline mutations, with no previous family history of the disease. We therefore analysed DNA from 21 women with sporadic LAM for TSC2 mutations. The sources of DNA were peripheral blood lymphocytes (12 patients), cultured lung cells obtained at the time of lung transplantation (eight patients), and angiomyolipoma (one patient).

Table 1 Presence or absence of angiomyolipomas in patient, and source of DNA for mutational analysis

\begin{tabular}{lll}
\hline Patient & $\begin{array}{l}\text { Angiomyolipoma(s) } \\
\text { present? }\end{array}$ & DNA source \\
\hline 421 & + & Peripheral blood lymphocytes \\
422 & - & Lymphoblastoid cell line \\
423 & + & Angiomyolipoma \\
476 & - & Peripheral blood lymphocytes \\
478 & - & Peripheral blood lymphocytes \\
480 & + & Lymphoblastoid cell line \\
481 & + & Lymphoblastoid cell line \\
489 & + & Lymphoblastoid cell line \\
491 & + & Lymphoblastoid cell line \\
492 & + & Lymphoblastoid cell line \\
494 & + & Lymphoblastoid cell line \\
496 & + & Lymphoblastoid cell line \\
505 & + & Lymphoblastoid cell line \\
537 & - & Cultured lung cells \\
538 & + & Cultured lung cells \\
539 & - & Cultured lung cells \\
540 & + & Cultured lung cells \\
541 & - & Cultured lung cells \\
542 & - & Cultured lung cells \\
543 & - & Cultured lung cells \\
547 & - & Cultured lung cells \\
\hline
\end{tabular}


A

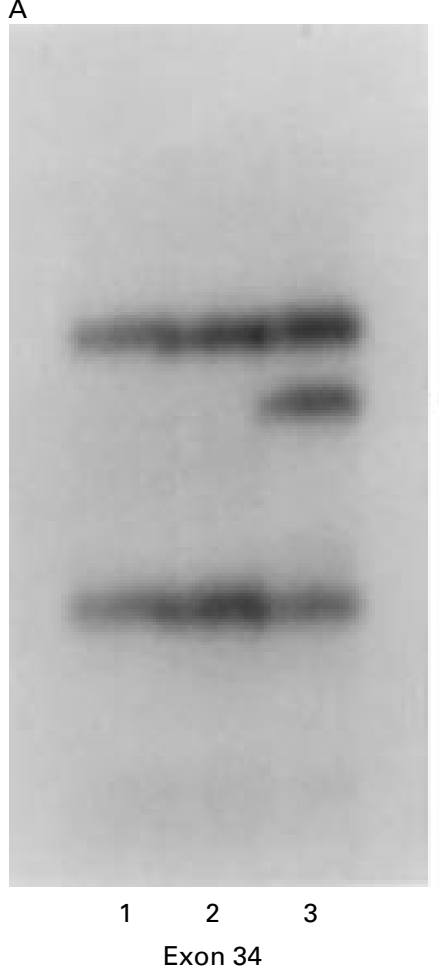

B

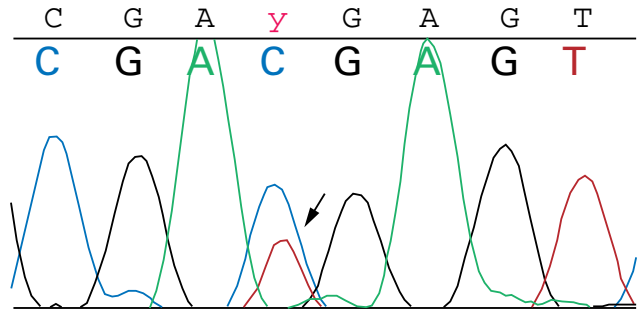

Figure 1 (A) Detection of variant band in exon 34. Lanes 1 and 2 contain DNA from controls and lane 3 contains DNA from patient 539. The arrow indicates the variant band. (B) Sequencing of exon 34 from patient 539. The arrow indicates the double peak of $C$ and $T$ at nucleotide 4536.

\section{Patients, methods, and results}

This study was approved by the Institutional Review Board of Fox Chase Cancer Center. None of the patients in this study had dermatological or neurological signs or symptoms of TSC. Twelve of the patients had known angiomyolipomas (table 1 ). The angiomyolipoma from patient 492 had TSC $2 \mathrm{LOH}^{12}$ The angiomyolipomas from patients 423, 480, 481, 489, and 491 did not have TSC1 or TSC2 LOH. ${ }^{12}$ Tissue from the remaining six angiomyolipomas was not available for $\mathrm{LOH}$ analysis. The source of DNA was either peripheral blood lymphocytes or a lymphoblastoid cell line for 12 patients (table 1). For one patient (423) DNA was isolated directly from fresh angiomyolipoma tissue. Fresh or frozen angiomyolipoma tissue from which genomic DNA could be prepared was not available from the other patients. For eight patients, DNA was isolated from primary cultures of lung tissue
Table 2 TSC2 polymorphisms in LAM patients

\begin{tabular}{llll}
\hline Patient & Exon & Nucleotide change & Amino acid \\
\hline 494 & 12 & C1281A & I427I \\
494 & 14 & C1578T & S526S \\
539 & 34 & C4536T & D1489D \\
\hline
\end{tabular}

established at the time of lung transplantation for LAM.

Single strand conformation analysis (SSCP) was used to search for mutations in the coding regions of the TSC2 gene. The primers amplifying each of the 41 exons of TSC2 and the PCR conditions have been previously reported by $\mathrm{Au}$ et al. ${ }^{13}$ The PCR products were run on MDE gels (AT Biochem). To maximise the detection of variant bands, each PCR product was run on two gels: one without glycerol and one with $5 \%$ glycerol. Samples in which variant bands were detected were reamplified and sequenced.

We found three variant bands in exons 12, 14, and 34, all of which were found by sequencing to represent silent polymorphisms (table 2). All three polymorphisms have been previously reported and are present in the TSC Variation Database (http://expmed. bwh.harvard.edu/ts/). No TSC2 alterations resulting in amino acid changes were detected.

\section{Discussion}

The clinical and pathological similarities between TSC and sporadic LAM have led to the hypothesis that LAM is a form of TSC. ${ }^{4}$ Our recent finding that $54 \%$ of angiomyolipomas from sporadic LAM patients have LOH in the TSC2 region of chromosome $16 \mathrm{p} 13^{12}$ supported this hypothesis. In this study, DNA samples from 21 women with pulmonary LAM, 12 of whom also had renal angiomyolipomas, were examined for TSC2 mutations in all 41 exons using SSCP. No definite mutations were identified.

Our data indicate that germline mutations in TSC2 are infrequent in sporadic LAM. In addition, the lack of TSC2 mutations in the eight DNA samples from primary cell cultures established from LAM lung tissue at the time of lung transplantation suggests that somatic mosaicism for TSC2 mutations is not a frequent cause of sporadic LAM. It is possible that in some patients, however, mutations were missed. The sensitivity of SSCP in TSC2 analysis is not known, but for other genes the sensitivity for a single gel condition has been estimated at $75-98 \% .{ }^{14}$ Each PCR product in our study was run under two gel conditions (with and without glycerol). We also did not screen for mutations in the non-coding regions of TSC 2 or for deletions. Large TSC 2 deletions are often associated with renal cysts, ${ }^{15}$ which were not present in any of the patients in this study. We did not analyse these samples for TSC1 mutations because we had previously found TSC2 LOH, but not TSC1 $\mathrm{LOH}$, in angiomyolipomas from women with sporadic LAM. ${ }^{12}$

In summary, this is the first report of TSC2 mutational analysis in patients with pulmonary lymphangiomyomatosis. We did not find TSC2 
mutations in DNA isolated from lymphocytes (12 patients), primary cultures of lung tissue (eight patients), or angiomyolipoma (one patient). We conclude that germline mutations in the coding regions of TSC 2 are uncommon in sporadic LAM despite the striking clinical and pathological overlap between these two diseases. Additional studies will be required to determine whether other types of TSC2 mutations not detected by SSCP occur in sporadic LAM, whether some patients have somatic mosaicism for TSC2 mutations, or whether mutations in other genes are involved.

We are grateful to William A Petri Sr and Drs Ann Petri, Becky Raftoginis, and Warren Kruger for critical review of the manuscript, and to Dr Alfred Knudson, Dr Frank McCormack, Ms Sue Byrnes, and The LAM Foundation for their ongoing support. We also thank the patients who contributed blood and tissue specimens for this research. This work was supported by Institutes of Health (HL 60746).

1 Sullivan EJ. Lymphangioleiomyomatosis. A review. Chest 1998;114:1689-703.

2 Kalassian KG, Doyle R, Kao P, Ruoss S, Raffin TA Lymphangioleiomyomatosis: new insights. Am f Respir Crit Care Med 1997;155:1183-6.

3 Castro M, Shepherd CW, Gomez MR, Lie JT, Ryu JH. Pulmonary tuberous sclerosis. Chest 1995;107:189-95.

4 Kerr LA, Blute ML, Ryu JH, Swensen SJ, Malek RS. Renal angiomyolipoma in association with pulmonary angiomyolipoma in association with pulmonary lymphangioleiomyomatosis:
rosis. Urology 1993;41:440-4.
5 Bernstein SM, Newell JD, Adamczyk D, Mortenson RL, King TE, Lynch DA. How common are renal angiomyolipomas in patients with pulmonary lymphangiomyomatosis. Am f Respir Crit Care Med 1995;152:2138-43.

6 Chu SC, Horiba K, Usuki J, et al. Comprehensive evaluation of 35 patients with lymphangioleiomyomatosis. Chest 1999;115:1041-52.

7 Bonetti F, Chiodera P. Lymphangioleiomyomatosis and tuberous sclerosis: where is the border? Eur Respir $\mathcal{F} 1996$; 9:399-401.

8 van Slegtenhorst $M$, de Hoogt R, Hermans C, et al. Identification of the tuberous sclerosis gene TSC1 on chromosome 9q34. Science 1997;277:805-8.

9 European Chromosome 16 Tuberous Sclerosis Consortium. Identification and characterization of the tuberous sclerosis gene on chromosome 16. Cell 1993;75:1305-15.

10 Henske EP, Scheithauer BW, Short MP, et al. Allelic loss is frequent in tuberous sclerosis kidney lesions but rare in brain lesions. Am f Hum Genet 1996;59:400-6.

11 Henske EP, Neumann HPH, Scheithauer BW, Herbst EW, Short MP, Kwiatkowski DJ. Loss of heterozygosity in the Short MP, Kwiatkowski DJ. Loss of heterozygosity in the
tuberous sclerosis (TSC2) region of chromosome band tuberous sclerosis (TSC2) region of chromosome band
$16 \mathrm{p} 13$ occurs in sporadic as well as TSC-associated renal angiomyolipomas. Genes Chrom Cancer 1995;13:295-8.

12 Smolarek TA, Wessner LL, McCormack FX, Mylet JC, Menon AG, Henske EP. Evidence that lymphangiomyomatosis is caused by TSC2 mutations: chromosome $16 \mathrm{p} 13$ loss of heterozygosity in angiomyolipomas and lymph nodes from women with lymphangiomyomatosis. $\mathrm{Am}$ f nodes from women with
Hum Genet 1998;62:810-5.

$13 \mathrm{Au} \mathrm{KS}$, Rodriguez JA, Finch JL, et al. Germ-line mutational analysis of the TSC2 gene in 90 tuberous sclerosis patients. Am F Hum Genet 1997;62:286-94.

14 Ravnik-Glavac M, Glavac D, Dean M. Sensitivity of singlestrand conformation polymorphism and heteroduplex method for mutation detection in the cystic fibrosis gene. Hum Mol Genet 1994;3:801-7.

15 Sampson JR, Maheshwar MM, Aspinwall R, et al. Renal cystic disease in tuberous sclerosis: role of the polycystic kidney disease 1 gene. Am f Hum Genet 1997;61:843-51. 